\title{
Association Between MicroRNAs Polymorphisms and Risk of Ischemic Stroke: A Meta-Analysis in Chinese Individuals
}

\author{
Chen-Xi Li ${ }^{1}$, Hong Weng ${ }^{2,3}$, Jun Zheng ${ }^{1}$, Zhi-He Feng ${ }^{1}$, Jian-Lin Ou ${ }^{1}$ and Wei-Jing Liao ${ }^{1 *}$ \\ ${ }^{1}$ Department of Rehabilitation Medicine, Zhongnan Hospital of Wuhan University, Wuhan, China, ${ }^{2}$ Center for Evidence-Based \\ and Translational Medicine, Zhongnan Hospital of Wuhan University, Wuhan, China, ${ }^{3}$ Department of Urology, Zhongnan \\ Hospital of Wuhan University, Wuhan, China
}

\section{OPEN ACCESS}

Edited by:

Aurel Popa-Wagner,

Department of Neurology, University

Hospital Essen, Germany

Reviewed by:

Hermona Soreq,

Hebrew University of Jerusalem, Israel

Petr A. Slominsky,

Institute of Molecular Genetics (RAS),

Russia

*Correspondence:

Wei-Jing Liao

weijingliao@sina.com

Received: 19 January 2018

Accepted: 12 March 2018

Published: 28 March 2018

Citation:

Li C-X, Weng H, Zheng J, Feng Z-H,

Ou J-L and Liao W-J (2018) Association Between MicroRNAs

Polymorphisms and Risk of Ischemic

Stroke: A Meta-Analysis in Chinese

Individuals.

Front. Aging Neurosci. 10:82. doi: 10.3389/fnagi.2018.00082
Objective: Previous studies have demonstrated that some single-nucleotide polymorphisms (SNPs) in miRNAs are related to the risk of ischemic stroke (IS), but the conclusions are still controversial and inconclusive. We performed this meta-analysis to further assess the association between miR-146a C>G (rs2910164), miR-149 T>C (rs2292832), miR-196a2 T>C (rs11614913), miR-499 A>G (rs3746444) and risk of IS in Chinese individuals.

Methods: Relevant studies were identified in the databases of PubMed, Embase. The strength of correlation between microRNAs polymorphisms and IS risk was assessed by odds ratios (ORs) and 95\% confidence intervals (95\% Cls) under five genetic models.

Results: 5 studies, containing 2,632 cases and 3,191 controls, were included in this meta-analysis. The overall results of meta-analysis indicated that there were no significant association between miR-146a $C>G$ (rs2910164), miR-149 T>C (rs2292832), miR-196a2 T>C (rs11614913), and the IS risk in the overall analyses. MiR-499 A>G (rs3746444) was associated with an increased IS risk under allele model $(\mathrm{OR}=1.30,95 \% \mathrm{Cl}=1.02-1.66)$, heterozygous model $(\mathrm{OR}=1.35,95 \%$ $\mathrm{Cl}=1.01-1.79)$ and dominant model $(\mathrm{OR}=1.36,95 \% \mathrm{Cl}=1.02-1.80)$ in Chinese. The sensitivity analysis results of these four polymorphisms were similar to the overall results.

Conclusion: MiR-499 A>G (rs3746444) G allele and AG, AG + AA genotype might be risk factors of IS in Chinese. No significant association was observed between miR-146a C>G (rs2910164), miR-149 T>C (rs2292832), miR-196a2 T>C (rs11614913), and IS risk. The associations may be different due to geographical factors of China. More explorations in more diverse geographically regions with large sample size are expected to further verify the findings in the future.

Keywords: miR-146a, miR-149, miR-196a2, miR-499, polymorphism, ischemic stroke, risk, meta-analysis

\section{INTRODUCTION}

Neurological disorders are the second leading cause of death and a major cause of functional disability worldwide, among which stroke accounted for the highest proportion, imposing a huge burden for the whole society as well as the healthcare system (GBD Neurological Disorders Collaborator Group, 2017). Ischemic stroke (IS) occupies approximately 85\% 
of all types of strokes (Kotlega et al., 2017). The risk factors of IS include hypertension, smoking, waist-to-hip ratio, diet, diabetes mellitus, etc. (O’Donnell et al., 2010). Moreover, a large range of genetic factors mingled with environmental factors stimulated the complex disease, and it is indicated that genetic ones play a significant role in the cause of IS (Boehme et al., 2017; Pan et al., 2017; Xiang et al., 2017).

MicroRNAs (miRNAs) are non-coding, endogenous $\sim 22 \mathrm{nt}$ RNAs, which can induce either translational repression or mRNA degradation, and regulate post-transcriptional gene expression by binding to the $3^{\prime}$-untranslated region ( $3^{\prime}$-UTR) of target genes (Bartel, 2009). The most abundant form of DNA variation in the human genome are single nucleotide polymorphisms (SNPs), which can potentially change various biological processes by affecting the maturation process or target selection of miRNAs (Duan et al., 2007). Previous studies have demonstrated that miR-146a G, miR-149 T, miR-196a2 C, miR-499 G alleles are possible genetic predisposing factors (Jeon et al., 2012; Min et al., 2012; Park et al., 2012; Wang et al., 2012). These four SNPs in miRNAs (miR-SNPs) can influence vascular damage responses, and regulate miRNA targets related to thrombosis and inflammation pathways in the circulation system (Luthra et al., 2008; El Gazzar et al., 2011; Yang et al., 2012; Wu et al., 2013). Among them, miR-146a is closely related to regulation of tumor necrosis factor- $\alpha$ (TNF- $\alpha$ ) (El Gazzar et al., 2011), meanwhile miR-149 can regulate the expression of MTHFR (Wu et al., 2013), miR-196a2 can target annexin A1 (Luthra et al., 2008), and miR499 can affect the inflammatory reaction through modulating C-reactive protein (CRP) (Yang et al., 2012) respectively. Several studies aimed to explore the influence of these four SNPs on IS risk, but the conclusions are still controversial and inconclusive. We performed this meta-analysis to further assess the association between miR-146a C > G (rs2910164), miR-149 $\mathrm{T}>\mathrm{C}(\mathrm{rs} 2292832), \operatorname{miR}-196 \mathrm{a} 2 \mathrm{~T}>\mathrm{C}(\mathrm{rs} 11614913), \mathrm{miR}-499 \mathrm{~A}>\mathrm{G}$ (rs3746444) and risk of IS in Chinese individuals, which may be likely to be utilized as clinical indexes to evaluate the risk of occurrence, development and responses to the treatments of IS.

\section{MATERIALS AND METHODS}

\section{Search Strategy}

We conducted a comprehensive literature search of PubMed, Embase up to November 30, 2017. The searched terms are as follows: ["ischemic stroke" AND ("microRNA" OR "miRNA" OR “miR") AND ("polymorphism" OR "SNP” OR "mutation" OR "variant")]. In order to identify other studies which may be relevant with this from references, all studies were retrieved in the manual way. We performed the meta-analysis according to Preferred Reporting Items for Systematic Reviews and MetaAnalyses (PRISMA) statement in reporting meta-analysis.

\section{Eligibility Criteria}

Eligible studies should conform with the following criteria: (1) the exposure was microRNAs polymorphisms; (2) populations were divided into case and control groups, consisting of IS and without IS respectively; (3) the outcome was incident of IS; (4) the study design was accordance with case-control study; (5) study provided sufficient published data to calculate the odds ratios (ORs) with corresponding 95\% confidence intervals (CIs). The exclusion conditions include: (1) repeated publications (studies recently published or with more participants were included); (2) abstract, reviews, meta-analysis, case reports and comments. (3) animal model research. (4) not about Chinese individuals; (5) not published in English.

\section{Data Extraction}

Information was extracted from all included studies for analysis, including first author's name, published year, country, ethnicity, source of controls, genotype frequency of case and control, age, genotyping method, and HWE for controls by two independent researchers (Li and Weng). Discrepancy was resolved by referring to original studies in discussion with a third reviewer (Zheng).

\section{Statistical Analysis}

Chi-square test was used to determine Hardy-Weinberg equilibrium (HWE) in the control groups, which could be considered disequilibrium when $P$ value is less than 0.05 . The strength of correlation between microRNAs polymorphisms and IS risk was assessed by ORs and 95\% confidence intervals (95\% CIs) under five genetic models. Heterogeneity between the studies was evaluated by both the Cochran's Q statistic and the $I^{2}$ statistic (Higgins, 2008). $P<0.10$ and/or $I^{2}>50 \%$ indicated significant heterogeneity, and random-effects model (the DerSimonian and Laird method) was applied for ORs calculation; otherwise, fixed-effects model using the MantelHaenszel was utilized. The sensitivity analysis was performed according to HWE status of controls (Thakkinstian et al., 2005). Furthermore, Begg's funnel plot and Egger's test were used to inspect potential publication bias among included studies. The statistical significance of the ORs was determined by $Z$-test, with $P<0.05$ suggesting a significant difference. All statistical analyses were conducted with the software Stata 14.0.

\section{RESULTS}

\section{Characteristics of Included Studies}

We yielded 85 papers initially and 5 studies, containing 2,632 cases and 3,191 controls, were included in this meta-analysis finally. Figure 1 shows the progress of study selection. Among these studies, five studies focused on miR-146a G>C (rs2910164) (Liu et al., 2014; Zhu et al., 2014; Huang et al., 2015; Qu et al., 2016; Luo et al., 2017), one study focused on miR-149 T>C (rs2292832) (Luo et al., 2017), four studies focused on miR-196a2 T>C (rs11614913) (Liu et al., 2014; Zhu et al., 2014; Huang et al., 2015; Luo et al., 2017) and three studies focused on miR-499 A > G (rs3746444) (Liu et al., 2014; Huang et al., 2015; Luo et al., 2017). The characteristics of the included studies are presented in Table 1.Among them, despite one study (Qu et al., 2016) in miR-146a G>C (rs2910164), one study (Huang et al., 2015) in miR-499 A > G (rs3746444), all conformed with HWE.

\section{Heterogeneity Test}

The result of between-study heterogeneity of genetic models of miR-146a G>C (rs2910164) polymorphism was significant 


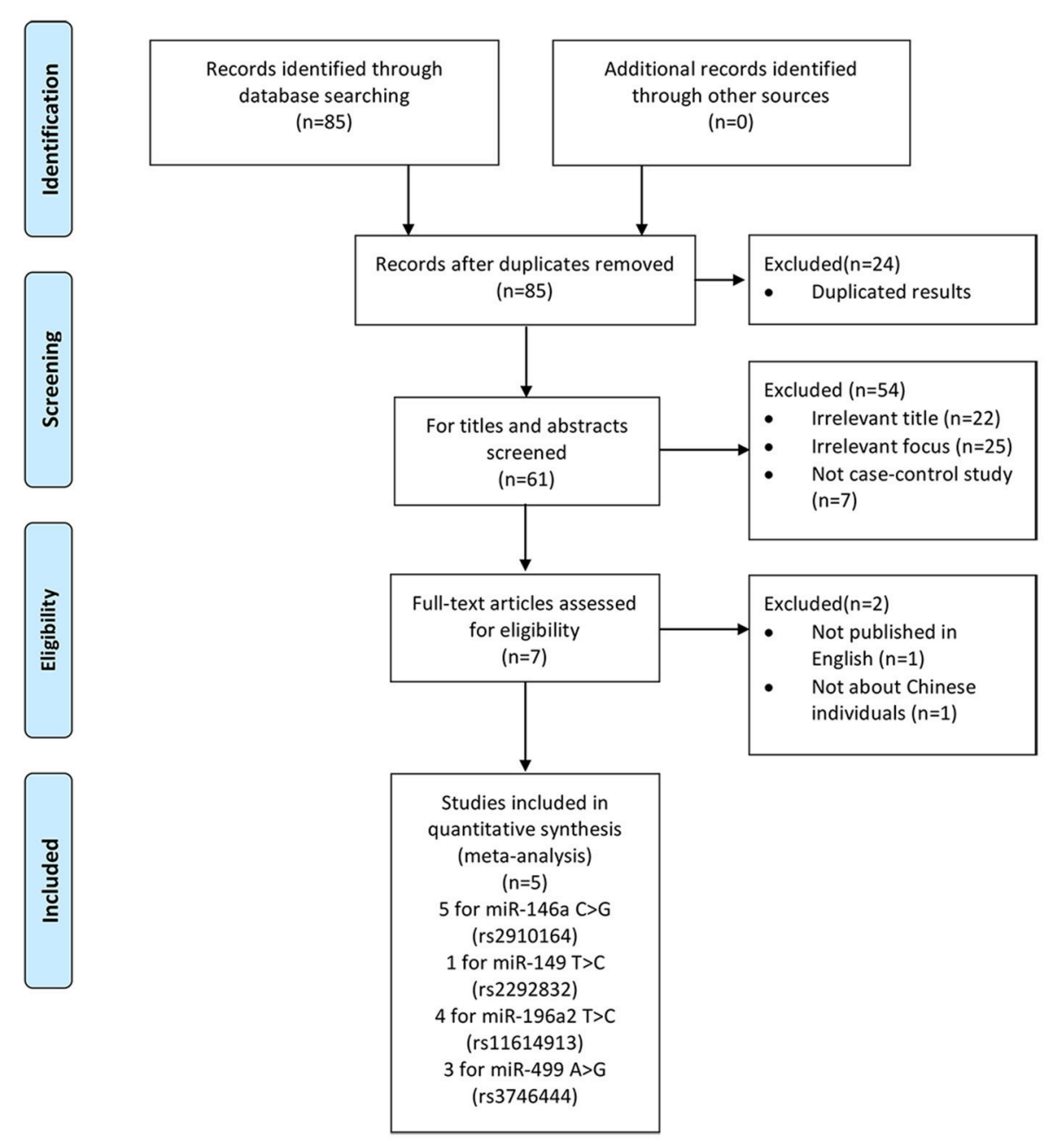

FIGURE 1 | Flow chart of study selection process.

(Table 2) under three models (G vs. C, $I^{2}=60.1, P=0.04$; GG vs. CC, $I^{2}=61.4, P=0.04$; GG vs. $\mathrm{CG}+\mathrm{CC}, I^{2}=50.2, P$ $=0.09$ ). The similar trend can be observed in miR-499 $\mathrm{A}>\mathrm{G}$ (rs3746444) polymorphism under allele model (G vs. A, $I^{2}=$ 52.8, $P=0.12$ ), heterozygous model (AG vs. AA, $I^{2}=54.3, P$ $=0.11$ ) and dominant model (AG + GG vs. AA, $I^{2}=56.1, P=$ 0.10 ), thus random-effects model was used for meta-analysis.

The fixed-effects model was used to calculate ORs for miR-196a2 T>C (rs11614913) polymorphism due to the low heterogeneity under all genetic models. No evidence of significant heterogeneity was detected in the heterozygous model (CG vs.CC, $\left.I^{2}=0, P=0.46\right)$ and dominant model (CG + GG vs. $\left.\mathrm{CC}, I^{2}=39.3, P=0.16\right)$ of miR-146a G $>C$ (rs2910164). So does the homozygous model (GG vs. AA, $I^{2}=18.6, P=0.27$ ) and recessive model (GG vs. $\mathrm{AG}+\mathrm{AA}, I^{2}=17.7, P=0.27$ ) of miR-499 A>G (rs3746444) polymorphism.

The vast territory of China consists of different climate zones as well as environmental differences are known to exist between the Northern and Southern, the coastal and inland, mountains and plains. The meta-analysis verified our result and also indicated that geographical factors may be one of the main causes of high heterogeneity.

\section{MiR-146a C > G (rs2910164) and the Risk of Is}

There was no significant association between this SNP and the IS risk in the overall analyses. ( $\mathrm{G}$ vs. $\mathrm{C}$ : $\mathrm{OR}=0.99,95 \% \mathrm{CI}=0.87-$ 1.12; GG vs. CC: $\mathrm{OR}=0.99,95 \% \mathrm{CI}=0.74-1.31$; $\mathrm{CG}$ vs. $\mathrm{CC}: \mathrm{OR}$ $=0.99 ; 95 \% \mathrm{CI}=0.88-1.11 ; \mathrm{CG}+\mathrm{GG}$ vs. $\mathrm{CC}: \mathrm{OR}=0.99,95 \% \mathrm{CI}$ $=0.89-1.11$; GG vs. CG + CC: $\mathrm{OR}=0.99,95 \% \mathrm{CI}=0.79-1.24$, Table 2).

The results of sensitivity analysis were similar to the overall analyses (Figure 2).

\section{MiR-149 T>C (rs2292832) and the Risk of Is}

No significant correlation was found in the overall analyses or sensitive analyses in any one of these models ( $\mathrm{C}$ vs. T: $\mathrm{OR}=0.88$, $95 \% \mathrm{CI}=0.70-1.12$; $\mathrm{CC}$ vs. $\mathrm{TT}: \mathrm{OR}=0.80,95 \% \mathrm{CI}=0.49-1.31$; 
TABLE 1 | Major characteristics of included studies.

\begin{tabular}{|c|c|c|c|c|c|c|c|c|}
\hline First author & Year & Country & Ethnicity & $\begin{array}{l}\text { Source of } \\
\text { control }\end{array}$ & $\begin{array}{l}\text { Age } \\
\text { (Case/Control) }\end{array}$ & $\begin{array}{l}\text { Sample size } \\
\text { (Case/Control) }\end{array}$ & $\begin{array}{l}\text { Genotyping } \\
\text { method }\end{array}$ & $\begin{array}{l}P \text { for } \\
\text { HWE }\end{array}$ \\
\hline \multicolumn{9}{|c|}{ miR-146a C> G (rs2910164) } \\
\hline Liu & 2014 & China & Asian & Healthy & $67.52 \pm 10.29 / 66.34 \pm 11.07$ & 296/391 & PCR-RFLP & 0.650 \\
\hline Zhu & 2014 & China & Asian & Healthy & $61.62 \pm 0.986 / 62.05 \pm 0.982$ & $368 / 381$ & PCR-LDR & 0.952 \\
\hline Huang & 2015 & China & Asian & Healthy & $63(54,70) / 61(54,68)$ & $531 / 531$ & Taqman & 0.107 \\
\hline Qu & 2016 & China & Asian & Healthy & $61.30 \pm 9.40 / 59.50 \pm 8.50$ & $1,139 / 1,585$ & PCR-LDR & $<0.01$ \\
\hline Luo & 2017 & China & Asian & Healthy & $60.70 \pm 12.33 / 60.17 \pm 10.32$ & $298 / 303$ & PCR & 0.672 \\
\hline \multicolumn{9}{|c|}{ miR-149 T>C (rs2292832) } \\
\hline Luo & 2017 & China & Asian & Healthy & $60.70 \pm 12.33 / 60.17 \pm 10.32$ & 298/303 & PCR & 0.447 \\
\hline \multicolumn{9}{|c|}{ miR-196a2 T>C (rs11614913) } \\
\hline Liu & 2014 & China & Asian & Healthy & $67.52 \pm 10.29 / 66.34 \pm 11.07$ & 296/391 & PCR-RFLP & 0.060 \\
\hline Zhu & 2014 & China & Asian & Healthy & $61.62 \pm 0.986 / 62.05 \pm 0.982$ & $368 / 381$ & PCR-LDR & 0.384 \\
\hline Huang & 2015 & China & Asian & Healthy & $63(54,70) / 61(54,68)$ & $531 / 531$ & Taqman & 0.856 \\
\hline Luo & 2017 & China & Asian & Healthy & $60.70 \pm 12.33 / 60.17 \pm 10.32$ & 298/303 & PCR & 0.385 \\
\hline \multicolumn{9}{|c|}{ miR-499 A>G (rs3746444) } \\
\hline Liu & 2014 & China & Asian & Healthy & $67.52 \pm 10.29 / 66.34 \pm 11.07$ & 296/391 & PCR-RFLP & 0.170 \\
\hline Huang & 2015 & China & Asian & Healthy & $63(54,70) / 61(54,68)$ & $531 / 531$ & Taqman & $<0.01$ \\
\hline Luo & 2017 & China & Asian & Healthy & $60.70 \pm 12.33 / 60.17 \pm 10.32$ & 298/303 & PCR & 0.132 \\
\hline
\end{tabular}

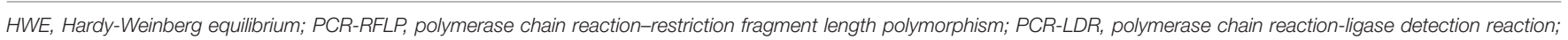
$P C R$, polymerase chain reaction.

TABLE 2 | Overall and sensitivity analyses of miR-146a C>G (rs2910164) and risk of IS.

\begin{tabular}{|c|c|c|c|c|c|c|c|}
\hline \multirow[t]{2}{*}{ Genetic model } & \multirow[t]{2}{*}{ Subgroup } & \multirow[t]{2}{*}{ No. of studies } & \multicolumn{2}{|c|}{ Meta-analysis } & \multirow[t]{2}{*}{$P$ for Egger's test } & \multicolumn{2}{|c|}{ Heterogeneity } \\
\hline & & & OR $(95 \% \mathrm{Cl})$ & $P$-value & & $I^{2}(\%)$ & $P$-value \\
\hline \multirow[t]{2}{*}{ G vs. C } & Overall & 5 & $0.99(0.87-1.12)$ & 0.87 & 0.77 & 60.1 & 0.04 \\
\hline & HWE-Yes & 4 & $0.99(0.82-1.19)$ & 0.88 & & 69.8 & 0.02 \\
\hline \multirow[t]{2}{*}{ GG vs. CC } & Overall & 5 & $0.99(0.74-1.31)$ & 0.92 & 0.85 & 61.4 & 0.04 \\
\hline & HWE-Yes & 4 & $0.98(0.65-1.48)$ & 0.94 & & 70.8 & 0.02 \\
\hline \multirow[t]{2}{*}{ CG vs. CC } & Overall & 5 & $0.99(0.88-1.11)$ & 0.85 & 0.50 & 0 & 0.46 \\
\hline & HWE-Yes & 4 & $1.01(0.86-1.18)$ & 0.93 & & 13.7 & 0.32 \\
\hline \multirow[t]{2}{*}{$\mathrm{CG}+\mathrm{GG}$ vs. CC } & Overall & 5 & $0.99(0.89-1.11)$ & 0.87 & 0.31 & 39.3 & 0.16 \\
\hline & HWE-Yes & 4 & $0.99(0.80-1.23)$ & 0.94 & & 53.5 & 0.09 \\
\hline \multirow[t]{2}{*}{ GG vs. $C G+C C$} & Overall & 5 & $0.99(0.79-1.24)$ & 0.94 & 0.85 & 50.2 & 0.09 \\
\hline & HWE-Yes & 4 & $0.99(0.71-1.37)$ & 0.94 & & 62.6 & 0.05 \\
\hline
\end{tabular}

OR, odds ratio; Cl, confidence interval; HWE, Hardy-Weinberg equilibrium.

TC vs. TT: $\mathrm{OR}=0.86 ; 95 \% \mathrm{CI}=0.61-1.22 ; \mathrm{TC}+\mathrm{CC}$ vs. TT: $\mathrm{OR}$ $=0.85,95 \% \mathrm{CI}=0.61-1.17 ; \mathrm{CC}$ vs. $\mathrm{TC}+\mathrm{TT}: \mathrm{OR}=0.87,95 \% \mathrm{CI}$ $=0.55-1.37$, Table 3, Figure 3).

\section{MiR-196a2 T>C (rs11614913) and the Risk of Is}

There was no significant association between this SNP and the IS susceptibility under all genetic models ( $\mathrm{C}$ vs. T: OR $=0.97,95 \%$ $\mathrm{CI}=0.88-1.07$; CC vs. TT: $\mathrm{OR}=0.93,95 \% \mathrm{CI}=0.76-1.15$; $\mathrm{TC}$ vs. TT: $\mathrm{OR}=0.97 ; 95 \% \mathrm{CI}=0.82-1.15 ; \mathrm{TC}+\mathrm{CC}$ vs. TT: $\mathrm{OR}=$
$0.96,95 \% \mathrm{CI}=0.82-1.13$; CC vs. TC + TT: OR $=0.96,95 \% \mathrm{CI}$ $=0.80-1.14$, Table 4, Figure 4).

MiR-499 A> G (rs3746444) and the Risk of Is The overall analysis showed that the miR-499 A>G (rs3746444) polymorphism increased the risk of IS under allele model ( $G$ vs. $\mathrm{A}, \mathrm{OR}=1.30,95 \% \mathrm{CI}=1.02-1.66$ ), heterozygous model (AG vs. $\mathrm{AA}, \mathrm{OR}=1.35 ; 95 \% \mathrm{CI}=1.01-1.79)$ and dominant model (AG + GG vs. AA: OR $=1.36,95 \% \mathrm{CI}=1.02-1.80)$. No significant association was observed between miR-499 A>G (rs3746444) polymorphism and risk of IS under homozygous model (GG vs. 


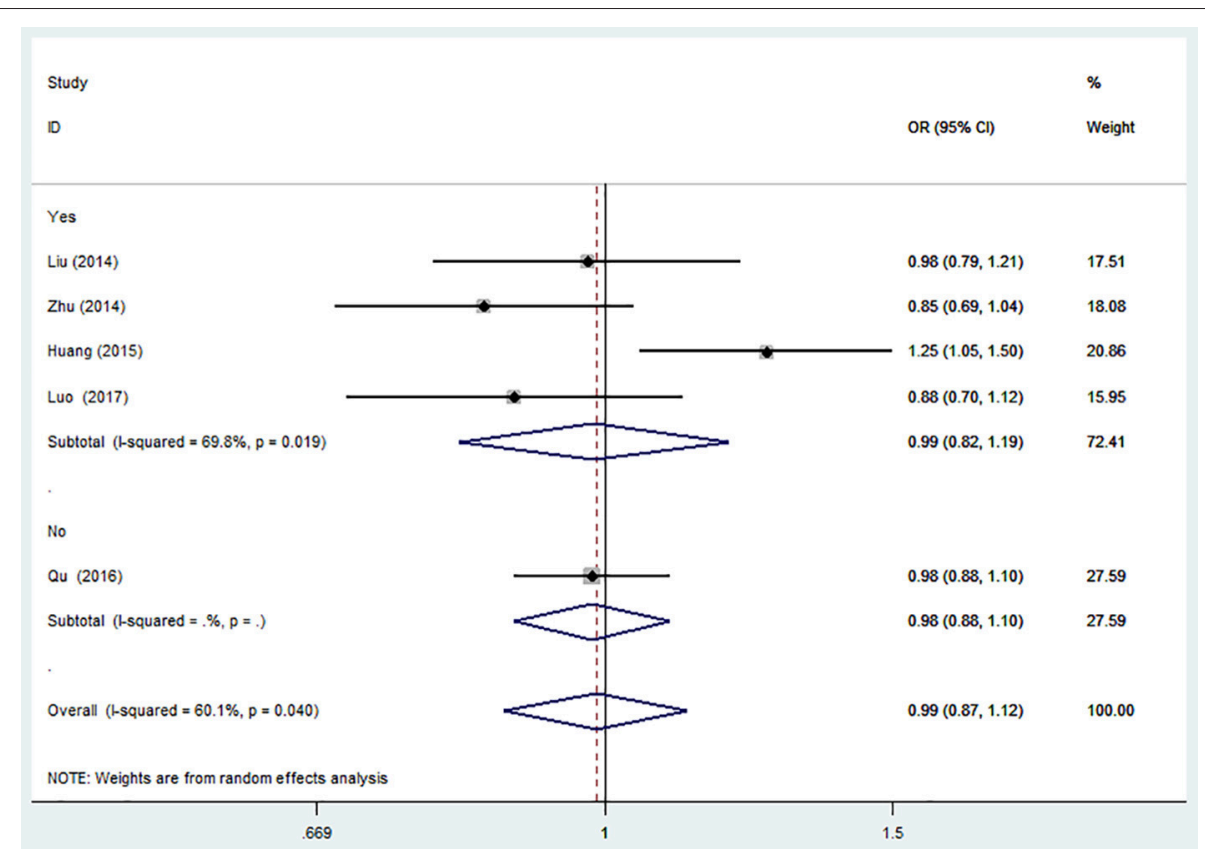

FIGURE 2 | Forest plot for the association between miR-146a C> G (rs2910164) polymorphism and ischemic stroke risk in allele genetic model.

TABLE 3 | Overall analyses of miR-149 T>C (rs2292832) and risk of IS.

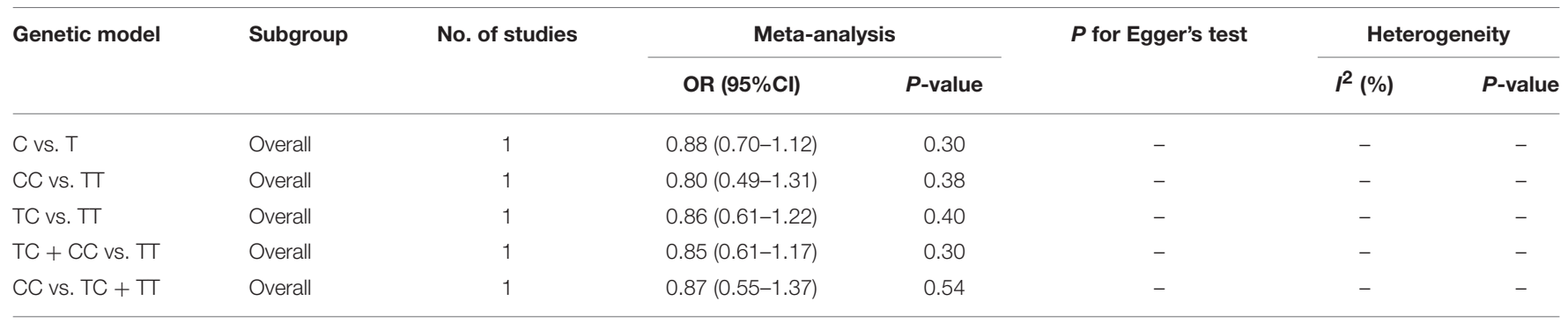

$\mathrm{OR}$, odds ratio; $\mathrm{Cl}$, confidence interval.

$\mathrm{AA}: \mathrm{OR}=1.02,95 \% \mathrm{CI}=0.71-1.46)$ and recessive model ( $\mathrm{GG}$ vs. $\mathrm{AG}+\mathrm{AA}, \mathrm{OR}=1.51,95 \% \mathrm{CI}=0.82-2.75)$ (Table 5, Figure 5).

Sensitivity analysis was conducted. However, no change of the result was detected.

\section{Publication Bias}

It is shown from Tables $\mathbf{2 - 5}$ that no significant publication bias was observed because the $P$-values for Egger's linear regression tests are no less than 0.05. Moreover, Begg's funnel plots did not show any significant asymmetry (Figure 6).

\section{DISCUSSION}

MiRNAs play key roles in many physiological and pathological processes, including tumorigenesis, proliferation, hematopoiesis, metabolism, immune function, epigenetics, and neurodegenerative diseases (Miska, 2005; O'Connell et al., 2010). SNP in miRNAs correlated closely with traditional risk factors of IS, such as atherosclerosis, hypertension, diabetes and hyperlipidemia (high cholesterol) (Boucherat et al., 2015; Li et al., 2015; Gómez-Banoy and Mockus, 2016; Schober and Weber, 2016). The four miRNAs we focused on-miR-146a, miR-149, miR-196a2, and miR-499-are intimately related to regulation of TNF- $\alpha$ (El Gazzar et al., 2011), MTHFR (Wu et al., 2013), annexin A1 (ANXA1) (Luthra et al., 2008), and CRP (Yang et al., 2012) respectively, which were general causes of cerebral ischemia. Above all, these miRNAs might be associated with risk of IS.

In the present meta-analysis, we found that the miR-499 A>G (rs3746444) G allele presented 1.24-fold higher risk of IS than A allele, as well as the AG and AG + GG carriers presented 1.35- and 1.36-fold higher risk than AA carriers, respectively. Previous researches have revealed that miR-499 involved in cell death under the anoxia and ischemia condition through its suppression of calcineurin-mediated dephosphorylation of dynamin-related protein-1 (Drp1), as a result the Drp1 accumulation in mitochondria and the Drp1-mediated activation of the mitochondrial fission machinery reduced (Wang et al., 


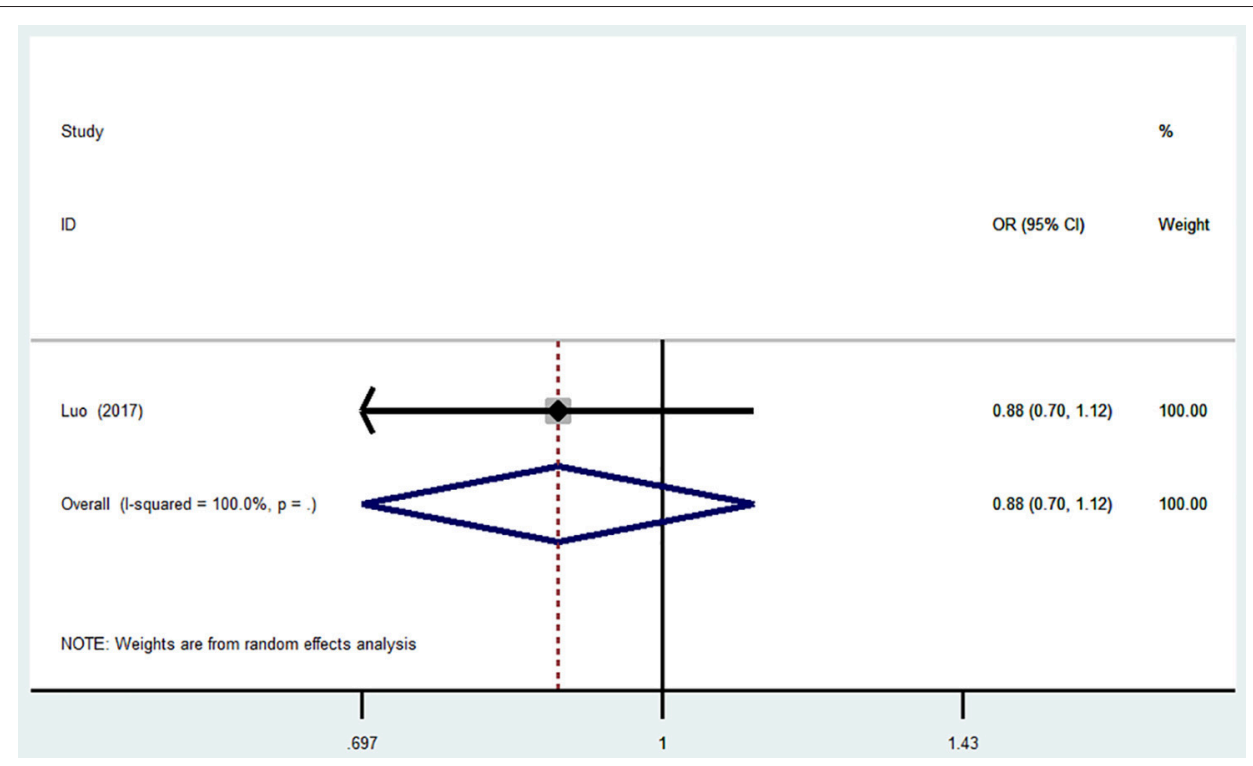

FIGURE 3 | Forest plot for the association between miR-149 T>C (rs2292832) polymorphism and ischemic stroke risk in allele genetic model.

TABLE 4 | Overall analyses of miR-196a2 T>C (rs11614913) and risk of IS.

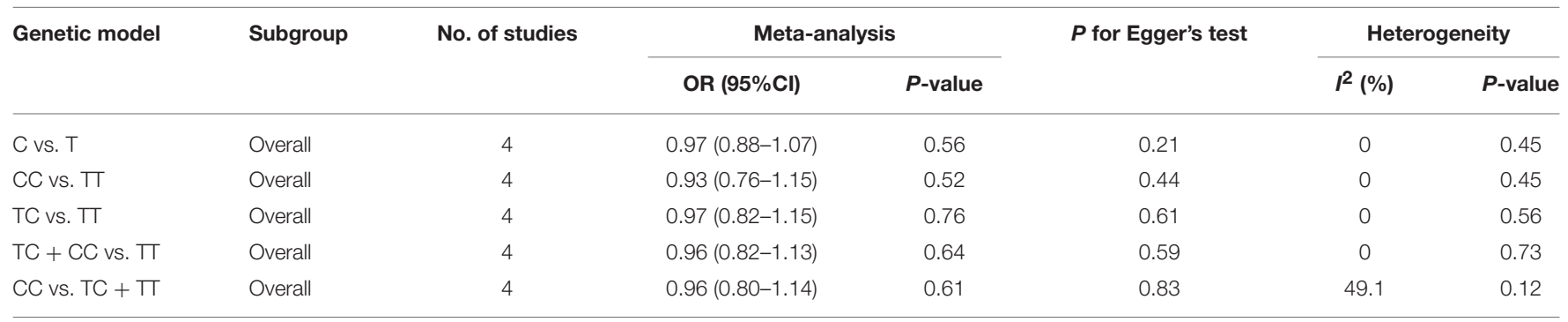

OR, odds ratio; $\mathrm{Cl}$, confidence interval.

2011). MiR-499 activates the production of CRP and regulates the expression of many inflammatory cytokines, including IL17RB, IL-23a, IL-2R, IL-6, IL-2 and IL-18R, so that it is able to influence the inflammatory reaction (Yang et al., 2012; Hashemi et al., 2013). Its increase of the concentration payed on serum or plasma CPR is likely to cause the increase of the incidence of stroke through regulating the level of risk factors of IS, such as in ammation, hypertension, and hyperlipoidemia (Luo et al., 2017). Liu et al. (2014) found the association between increased IS risk and rs3746444 A/G variant genotypes ( $G G+A G)$ was more notable in younger subjects, never smokers, non-obesity $(\mathrm{BMI} \leq 24)$, non-hypertension non-diabetes, non-coronary heart disease and non-hyperlipidemia. The results are inconsistent and its other potential mechanisms are still uncertain.

There was no significant association between miR-146a C>G (rs2910164) and IS risk in the overall analyses. According to the previous studies, miR-146a inhibits interleukin-1 receptorassociated kinase 1 (IRAK-1) and tumor necrosis factor receptor associated factor 6 (TRAF-6) expression through negative feedback regulation (Ramkaran et al., 2014), causing decreased levels of pro-inflammatory cytokines and critical transcription factor in atherosclerosis such as IL-1, IL-6, IL-8, TNF- $\alpha$, and resulting inhibition of nuclear factor $(\mathrm{NF})-\kappa \mathrm{B}$ via the Tolllike receptor pathway (Guo et al., 2010). Therefore, the downregulation of miR-146a tends to reduce inflammation-related atherosclerosis and have an effect on vascular damage response. The polymorphisms of miRNA will affect the further processing of pre-miRNA into a mature miRNA, therefore influence occurrence and development of disease (Saunders et al., 2007). Furthermore, miR-146a G allele may decrease the level of mature miRNA expression (Shen et al., 2008). Zhu et al. (2014) found that miR-146a CC genotype and C allele are associated with an increased incidence of large-artery atherosclerotic stroke in the northern Chinese Han population. However, Qu et al. (2016) conducted a study with 1,311 patients in six provinces and found rs2910164 has no association with IS incidence under several genetic models, stroke subtype and sex. It appears to be a strong predictor of stroke prognosis but not stroke incidence. Further reasons for the different results may include: the difference of geographical factors, sample size, study design, selection criteria of the patients or the diversity of genotyping methods, as well as not all studies obtained their results after 


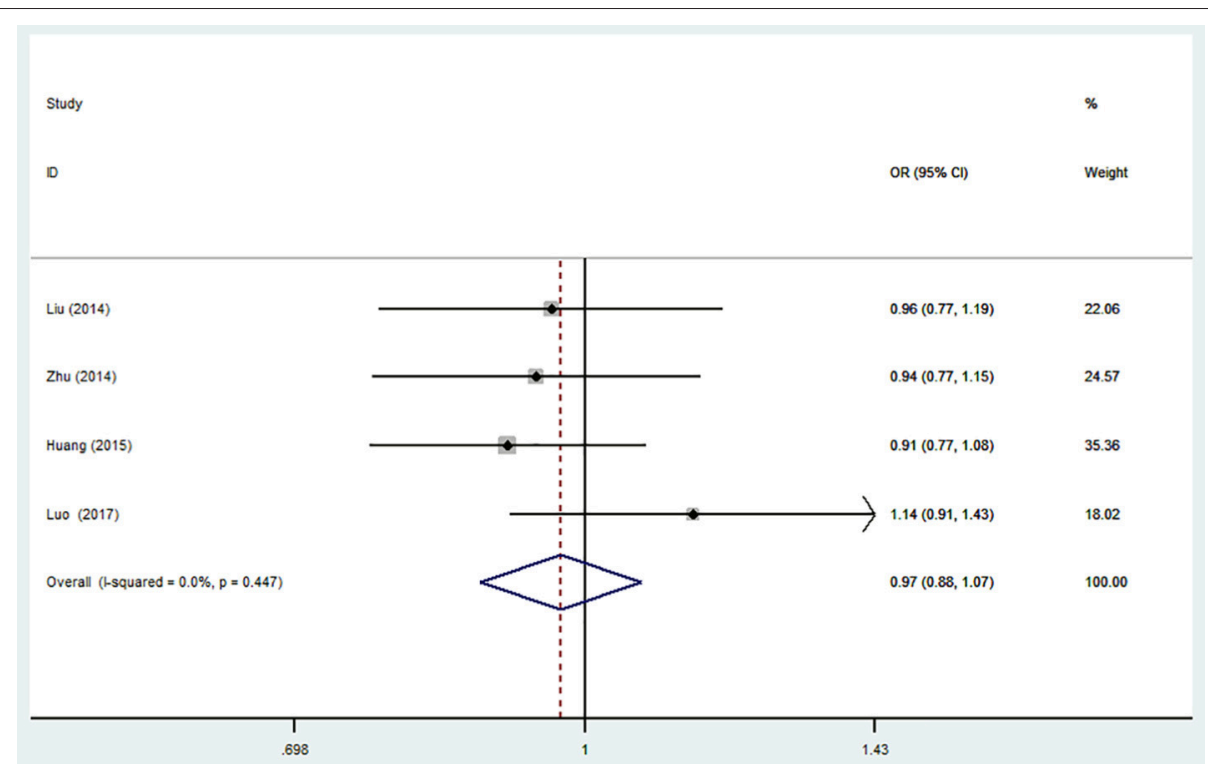

FIGURE 4 | Forest plot for the association between miR-196a2 T>C (rs11614913) polymorphism and ischemic stroke risk in allele genetic model.

TABLE 5 | Overall and sensitivity analyses of miR-499 A>G (rs3746444) and risk of IS.

\begin{tabular}{|c|c|c|c|c|c|c|c|}
\hline \multirow[t]{2}{*}{ Genetic model } & \multirow[t]{2}{*}{ Subgroup } & \multirow[t]{2}{*}{ No. of studies } & \multicolumn{2}{|c|}{ Meta-analysis } & \multirow[t]{2}{*}{$P$ for Egger's test } & \multicolumn{2}{|c|}{ Heterogeneity } \\
\hline & & & OR $(95 \% \mathrm{Cl})$ & $P$-value & & $I^{2}(\%)$ & $P$-value \\
\hline \multirow[t]{2}{*}{ G vs. A } & Overall & 3 & $1.30(1.02-1.66)$ & $<0.05$ & 0.67 & 52.8 & 0.12 \\
\hline & HWE-Yes & 2 & $1.48(1.20-1.83)$ & $<0.05$ & & 0 & 0.84 \\
\hline \multirow[t]{2}{*}{ GG vs. AA } & Overall & 3 & 1.69 (0.92-3.10) & 0.09 & - & 18.6 & 0.27 \\
\hline & HWE-Yes & 2 & 1.69 (0.92-3.10) & 0.09 & & 18.6 & 0.27 \\
\hline \multirow[t]{2}{*}{ AG vs. AA } & Overall & 3 & 1.35 (1.01-1.79) & $<0.05$ & 0.17 & 54.3 & 0.11 \\
\hline & HWE-Yes & 2 & $1.56(1.21-2.02)$ & $<0.05$ & & 0 & 0.67 \\
\hline \multirow[t]{2}{*}{$A G+G G$ vs. $A A$} & Overall & 3 & $1.36(1.02-1.80)$ & $<0.05$ & 0.36 & 56.1 & 0.10 \\
\hline & HWE-Yes & 2 & 1.58 (1.23-2.02) & $<0.05$ & & 0 & 0.93 \\
\hline \multirow[t]{2}{*}{ GG vs. $A G+A A$} & Overall & 3 & $1.51(0.82-2.75)$ & 0.19 & - & 17.7 & 0.27 \\
\hline & HWE-Yes & 2 & $1.51(0.82-2.75)$ & 0.19 & & 17.7 & 0.27 \\
\hline
\end{tabular}

OR, odds ratio; Cl, confidence interval; HWE, Hardy-Weinberg equilibrium.

adjustment of conflicting factors. More explorations in more diverse geographically regions with large sample size are expected to confirm the conclusions.

The present meta-analysis did not find association between miR-149 T>C (rs2292832), miR-196a2 T>C (rs11614913), and IS risk under all genetic models. In fact, no study has elucidated that the genetic polymorphism of miR-196a2 T $>C$ (rs11614913) is associated with stroke. For miR-149 T $>C$ (rs2292832), it might be spurious result because the results were on basis of only one study with limited participants. Whether miR-149 $\mathrm{T}>\mathrm{C}$ (rs2292832), miR-196a2 T>C (rs11614913), are related to IS risk or not is an interesting question for future investigation. Furthermore, the two SNPs mentioned have been demonstrated to be related to other human diseases, including cancer ( $\mathrm{Hu}$ et al., 2008), congenital heart disease (Xu et al., 2009) and coronary heart disease (Sung et al., 2016), or correlated with IS when they are combined with other SNPs, suggesting that these polymorphisms tend to influence different human diseases in various ways as well as reflecting different genetic or etiological factors for different diseases.

In addition, some studies explored the gene-gene, geneenvironment interaction between miRNA polymorphism and IS risk. Jeon et al. (2013) constructed all possible allele combinations and found miR-146a G/-149 T/-196a2 C/-499G, miR-146a C/-196a2 T/-499 A, miR-146a C/-196a2 T were significantly associated with disease prevalence in Korea. Huang et al. (2015) 

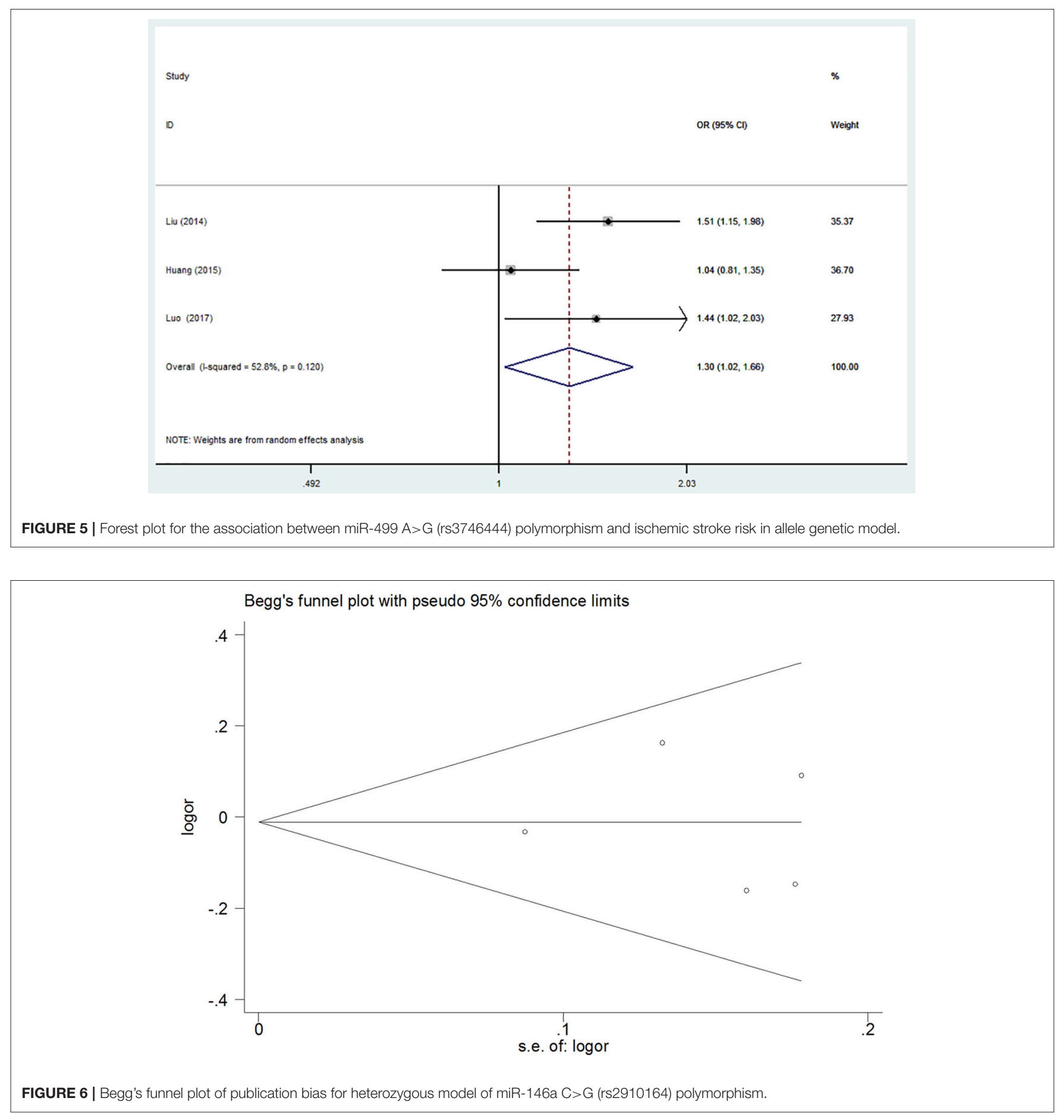

focused on the combined effects between the stroke-associated polymorphism (miR-146a) and fasting glucose, HDL-c, and LDLc levels on the risk of IS and found that the combined effects between miRNA polymorphism and fasting glucose/blood lipid levels may contribute to stroke pathogenesis.

To our knowledge, this is the first meta-analysis that evaluates the association between these four polymorphisms and risk of IS in Chinese. Sensitivity analysis by HWE status of controls made it more precise. Therefore, our findings were more reliable than the conclusions of previous studies. These results might provide further implications for evaluating risk of IS.

However, the limitations in our meta-analysis shouldn't be ignored when interpreting our findings. First of all, the evidence of between study heterogeneity was apparent, which might distort the conclusion of this meta-analysis. Second, the objects in all included studies were totally enrolled from hospitals, 
which might not be sufficient to represent the huge Chinese population and widespread distribution nationwide. Thirdly, IS is a complicated and multifactorial disease influenced by both genetic and environment factors. But the effects of the combination of genetic and environmental factors are difficult to be detected due to the lack of the accurate individual information in studies included. Lastly, a SNP might be in linkage disequilibrium with other genetic variations of stroke susceptibility genes, which may present stronger effect when considered together with other variations.

In conclusion, miR-499 $A>G$ (rs3746444) $G$ allele and $A G$, $\mathrm{AG}+\mathrm{AA}$ genotype might be risk factors of IS in Chinese. No significant association was observed between miR-146a C > G (rs2910164), miR-149 T>C (rs2292832), miR-196a2 T>C (rs11614913) polymorphism and risk of IS. The associations may be different due to geographical factors of China. More

\section{REFERENCES}

Bartel, D. P. (2009). MicroRNA target recognition and regulatory functions. Cell 136, 215-233. doi: 10.1016/j.cell.2009.01.002

Boehme, A. K., Esenwa, C., and Elkind, M., S. (2017). Stroke risk factors, genetics, and prevention. Circ. Res. 120, 472-495. doi: 10.1161/CIRCRESAHA.116.308398

Boucherat, O., Potus, F., and Bonnet, S. (2015). "MicroRNA and pulmonary hypertension," in microRNA: Medical Evidence: From Molecular Biology to Clinical Practice, ed G. Santulli (Cham: Springer International Publishing), 237-252.

Duan, R., Pak, C., and Jin, P. (2007). Single nucleotide polymorphism associated with mature miR-125a alters the processing of pri-miRNA. Hum. Mol. Genet. 16, 1124-1131. doi: $10.1093 / \mathrm{hmg} / \mathrm{ddm} 062$

El Gazzar, M., Church, A., Liu, T., and McCall, C. (2011). MicroRNA-146a regulates both transcription silencing and translation disruption of TNF$\alpha$ during TLR4-induced gene reprogramming. J. Leukoc. Biol. 90, 509-519. doi: $10.1189 /$ jlb.0211074

GBD Neurological Disorders Collaborator Group (2017). Global, regional, and national burden of neurological disorders during 1990-2015: a systematic analysis for the Global Burden of Disease Study 2015. Lancet Neurol. 16, 877-897. doi: 10.1016/S1474-4422(17)30299-5

Gómez-Banoy, N., and Mockus, I. (2016). [MicroRNAs: circulating biomarkers in type 2 Diabetes Mellitus and physical exercise]. Rev. Med. Chil. 144, 355-363. doi: $10.4067 / S 0034-98872016000300011$

Guo, M., Mao, X., Ji, Q., Lang, M., Li, S., Peng, Y., et al. (2010). MiR-146a in PBMCs modulates Th1 function in patients with acute coronary syndrome. Immunol. Cell Biol. 88, 555-564. doi: 10.1038/icb.2010.16

Hashemi, M., Eskandari-Nasab, E., Zakeri, Z., Atabaki, M., Bahari, G., Jahantigh, M., et al. (2013). Association of pre-miRNA-146a rs2910164 and pre-miRNA499 rs3746444 polymorphisms and susceptibility to rheumatoid arthritis. Mol. Med. Rep. 7, 287-291. doi: 10.3892/mmr.2012.1176

Higgins, J. (2008). Commentary: heterogeneity in meta-analysis should be expected and appropriately quantified. Int. J. Epidemiol. 37, 1158-1160. doi: $10.1093 / \mathrm{ije} / \mathrm{dyn} 204$

Hu, Z., Chen, J., Tian, T., Zhou, X., Gu, H., Xu, L., et al. (2008). Genetic variants of miRNA sequences and non-small cell lung cancer survival. J. Clin. Invest. 118, 2600-2608. doi: 10.1172/JCI34934

Huang, S., Zhou, S., Zhang, Y., Lv, Z., Li, S., Xie, C., et al. (2015). Association of the genetic polymorphisms in pre-MicroRNAs with risk of ischemic stroke in a Chinese population. PLOS ONE 10:e0117007. doi: 10.1371/journal.pone.0117007

Jeon, Y. J., Choi, Y. S., Rah, H., Kim, S. Y., Choi, D. H., Cha, S. H., et al. (2012). Association study of microRNA polymorphisms with risk of idiopathic explorations in more diverse geographically regions with large sample size are expected to further verify the findings in the future.

\section{AUTHOR CONTRIBUTIONS}

W-JL and C-XL: planned and designed the study; HW, C-XL, and JZ: searched the databases and extracted the data; C-XL, HW, and Z-HF: performed statistical analysis; C-XL: drafted the manuscript; W-JL and J-LO: reviewed the manuscript. All authors approved the final paper for submission and publication.

\section{ACKNOWLEDGMENTS}

We thank Yi-Guang Liu for his effort to polish this manuscript. recurrent spontaneous abortion in Korean women. Gene 494, 168-173. doi: 10.1016/j.gene.2011.12.026

Jeon, Y. J., Kim, O. J., Kim, S., Oh, S. H., Oh, D., Kim, O. J., et al. (2013). Association of the miR-146a, miR-149, miR-196a2, and miR-499 polymorphisms with ischemic stroke and silent brain infarction risk. Arterioscler. Thromb. Vasc. Biol. 33, 420-430. doi: 10.1161/ATVBAHA.112.300251

Kotlega, D., Peda, B., Zembron-Łacny, A., Gołab-Janowska, M., and Nowacki, P. (2017). The role of brain-derived neurotrophic factor and its single nucleotide polymorphisms in stroke patients. Neurol. Neurochir. Pol. 51, 240-246. doi: 10.1016/j.pjnns.2017.02.008

Li, T., Yang, G. M., Zhu, Y., Wu, Y., Chen, X. Y., Lan, D., et al. (2015). Diabetes and hyperlipidemia induce dysfunction of VSMCs: contribution of the metabolic inflammation/miRNA pathway. Am. J. Physiol. Endocrinol. Metab. 308, E257E269. doi: 10.1152/ajpendo.00348.2014

Liu, Y., Ma, Y., Zhang, B., Wang, S., Wang, X., and Yu, J. (2014). Genetic Polymorphisms in pre-microRNAs and risk of ischemic stroke in a Chinese population. J. Mol. Neurosci. 52, 473-480. doi: 10.1007/s12031-013-0152-Z

Luo, H. C., Luo, Q. S., Wang, C. F., Lei, M., Li, B. L., and Wei, Y. S. (2017). Association of miR-146a, miR-149, miR-196a2, miR-499 gene polymorphisms with ischemic stroke in a Chinese people. Oncotarget 8, 81295-81304. doi: 10.18632/oncotarget.18333

Luthra, R., Singh, R. R., Luthra, M. G., Li, Y. X., Hannah, C., Romans, A. M., et al. (2008). MicroRNA-196a targets annexin A1: a microRNA-mediated mechanism of annexin A1 downregulation in cancers. Oncogene 27, 6667-6678. doi: 10.1038/onc.2008.256

Min, K. T., Kim, J. W., Jeon, Y. J., Jang, M. J., Chong, S. Y., Oh, D., et al. (2012). Association of the miR-146aC>G, 149C>T, 196a2C $>$ T, and $499 \mathrm{~A}>\mathrm{G}$ polymorphisms with colorectal cancer in the Korean population. Mol. Carcinog. 51(Suppl. 1), E65-E73. doi: 10.1002/mc.21849

Miska, E. (2005). How microRNAs control cell division, differentiation and death. Curr. Opin. Genet. Dev. 15, 563-568. doi: 10.1016/j.gde.2005.08.005

O'Connell, R., Rao, D., Chaudhuri, A., and Baltimore, D. (2010). Physiological and pathological roles for microRNAs in the immune system. Nat. Rev. Immunol. 10, 111-122. doi: 10.1038/nri2708

O’Donnell, M. J., Xavier, D., Liu, L., Zhang, H., Chin, S. L., Rao-Melacini, P., et al. (2010). Risk factors for ischaemic and intracerebral haemorrhagic stroke in 22 countries (the INTERSTROKE study): a case-control study. Lancet 376, 112-123. doi: 10.1016/S0140-6736(10)60834-3

Pan, Y., Chen, W., Xu, Y., Yi, X., Han, Y., Yang, Q., et al. (2017). Genetic polymorphisms and clopidogrel efficacy for acute ischemic stroke or transient ischemic attack: a systematic review and meta-analysis. Circulation 135, 21-33. doi: 10.1161/CIRCULATIONAHA.116.024913

Park, Y. S., Jeon, Y. J., Lee, B. E., Kim, T. G., Choi, J. U., Kim, D. S., et al. (2012). Association of the miR-146aC $>$ G, miR-196a2C $>$ T, and miR-499A $>$ G 
polymorphisms with moyamoya disease in the Korean population. Neurosci. Lett. 521, 71-75. doi: 10.1016/j.neulet.2012.05.062

Qu, J. Y., Xi, J., Zhang, Y. H., Zhang, C. N., Song, L., Song, Y., et al. (2016). Association of the MicroRNA-146a SNP rs2910164 with ischemic stroke incidence and prognosis in a Chinese population. Int. J. Mol. Sci. 17:E660. doi: 10.3390/ijms17050660

Ramkaran, P., Khan, S., Phulukdaree, A., Moodley, D., and Chuturgoon, A. (2014). MiR-146a polymorphism influences levels of miR-146a, IRAK-1, and TRAF6 in young patients with coronary artery disease. Cell Biochem. Biophys. 68, 259-266. doi: 10.1007/s12013-013-9704-7

Saunders, M. A., Liang, H., and Li, W.-H. (2007). Human polymorphism at microRNAs and microRNA target sites. Proc. Natl. Acad. Sci. U.S.A. 104, 3300-3305. doi: 10.1073/pnas.0611347104

Schober, A., and Weber, C. (2016). Mechanisms of MicroRNAs in atherosclerosis. Annu. Rev. Pathol. 11, 583-616. doi: 10.1146/annurev-pathol-012615-044135

Shen, J., Ambrosone, C. B., DiCioccio, R. A., Odunsi, K., Lele, S. B, and Zhao, H. (2008). A functional polymorphism in the miR-146a gene and age of familial breast/ovarian cancer diagnosis. Carcinogenesis 29, 1963-1966. doi: 10.1093/carcin/bgn172

Sung, J., Kim, S., Yang, W., Kim, W., Moon, J., Kim, I., et al. (2016). MiRNA polymorphisms (miR-146a, miR-149, miR-196a2 and miR-499) are associated with the risk of coronary artery disease. Mol. Med. Rep. 14, 2328-2342. doi: 10.3892/mmr.2016.5495

Thakkinstian, A., McElduff, P., D’Este, C., Duffy, D., and Attia, J. (2005). A method for meta-analysis of molecular association studies. Stat. Med. 24, 1291-1306. doi: 10.1002/sim.2010

Wang, A. X., Xu, B., Tong, N., Chen, S. Q., Yang, Y., Zhang, X. W., et al. (2012). Meta-analysis confirms that a common G/C variant in the pre-miR-146a gene contributes to cancer susceptibility and that ethnicity, gender and smoking status are risk factors. Genet. Mol. Res. 11, 3051-3062. doi: 10.4238/2012.August.31.2

Wang, J. X., Jiao, J. Q., Li, Q., Long, B., Wang, K., Liu, J. P., et al. (2011). MiR499 regulates mitochondrial dynamics by targeting calcineurin and dynaminrelated protein-1. Nat. Med. 17, 71-78. doi: 10.1038/nm.2282
Wu, C., Gong, Y., Sun, A., Zhang, Y., Zhang, C., Zhang, W., et al. (2013). The human MTHFR rs4846049 polymorphism increases coronary heart disease risk through modifying miRNA binding. Nutr. Metab. Cardiovasc. Dis. 23, 693-698. doi: 10.1016/j.numecd.2012. 02.009

Xiang, Y. F., Guo, J., Peng, Y., Tan, T., Huang, H., Luo, H. T., et al. (2017). Association of miR-21, miR-126 and miR-605 gene polymorphisms with ischemic stroke risk. Oncotarget 8, 95755-95763. doi: 10.18632/oncotarget. 21316

Xu, J., Hu, Z., Xu, Z., Gu, H., Yi, L., and Cao, H. (2009). Functional variant in microRNA-196a2 contributes to the susceptibility of congenital heart disease in a Chinese population. Hum. Mutat. 30, 1231-1236. doi: 10.1002/ humu.21044

Yang, B., Chen, J., Li, Y., Zhang, J., Li, D., Huang, Z., et al. (2012). Association of polymorphisms in pre-miRNA with inflammatory biomarkers in rheumatoid arthritis in the Chinese Han population. Hum. Immunol. 73, 101-106. doi: 10.1016/j.humimm.2011. 10.005

Zhu, R., Liu, X., He, Z., and Li, Q. (2014). MiR-146a and miR-196a2 polymorphisms in patients with ischemic stroke in the northern Chinese Han population. Neurochem. Res. 39, 1709-1716. doi: 10.1007/s11064-0141364-5

Conflict of Interest Statement: The authors declare that the research was conducted in the absence of any commercial or financial relationships that could be construed as a potential conflict of interest.

Copyright (c) $2018 \mathrm{Li}$, Weng, Zheng, Feng, Ou and Liao. This is an open-access article distributed under the terms of the Creative Commons Attribution License (CC $B Y)$. The use, distribution or reproduction in other forums is permitted, provided the original author(s) and the copyright owner are credited and that the original publication in this journal is cited, in accordance with accepted academic practice. No use, distribution or reproduction is permitted which does not comply with these terms. 